\title{
Hydrogen effect on the sigma-phase in $\mathrm{Fe}_{53.8} \mathrm{Cr}_{46.2}$
}

\author{
J. Cieslak ${ }^{\text {a }}$, B.F.O. Costa ${ }^{\text {b }}$, S.M. Dubiel ${ }^{\mathrm{a}}$, D. Fruchart ${ }^{\mathrm{c}}$, \\ N.E. Skryabina ${ }^{\mathrm{d}, *}$ \\ ${ }^{a}$ Faculty of Physics \& Applied Computer Science, AGH University of Science \& Technology, \\ al. Mickiewicza 30, 30059 Krakow, Poland \\ ${ }^{\mathrm{b}}$ CEMDRX, Physics Department, University of Coimbra, P-3004-516 Coimbra, Portugal \\ ' Institut Néel, CNRS, Département MCMF, Groupe IICE, B.P. 166, 38042 Grenoble Cedex 9, France \\ ${ }^{d}$ Department of Physics, Perm State University, Bukirev 15, 614990 Perm, Russia
}

Received 4 October 2007; received in revised form 29 November 2007; accepted 30 November 2007

\begin{abstract}
Investigation of the stability and properties of a sigma-phase type compound $\mathrm{Fe}_{53.8} \mathrm{Cr}_{46.2}$ after electrochemical hydrogen treatment was made using X-ray diffraction and ${ }^{57} \mathrm{Fe}$ Mössbauer spectroscopy techniques. The amount of the hydrogen uptake was estimated to be equal to $\sim 1.2 \mathrm{H}$ per unit formula, and its presence leads to an anisotropic cell parameter expansion. However, the hydrogenated state appears as metastable with reference to the aged samples analyzed a few days to months after loading with hydrogen.
\end{abstract}

(C) 2008 Published by Elsevier B.V.

PACS: 75.40.-s; 71.20.LP

Keywords: $\mathrm{Fe}-\mathrm{Cr}$ alloy; Metal hydride; Structure transformation

\section{Introduction}

For both theoretical and technological aspects a large interest is still devoted to metal-hydrogen systems. Early, it was pointed out that hydrogenation of pure metals and alloys causes marked change of many properties, e.g. mechanical ones, first in the solid solution regime, and furthermore after the hydride phase formation. We have investigated hydrogenation issue in different classes of alloys - from solid solution to metal hydride transitions - either having martensite phase transformation (such as TiNi-type alloys) [1], or exhibiting short range atom ordering such as amorphous to nanocrystalline materials [2]. With this respect, $\mathrm{Fe}-\mathrm{Cr}$ alloys with $\sigma$-phase type structure form a class of materials interesting to consider for hydrogen treatment. In fact, the $\sigma$-phases have retained much interest since they appear in technologically important materials, e.g. heat resistant steels, as brittle precipitates, and often drastically deteriorate their useful properties. Concerning a criterion of stability, the valence electron number per atom is a relevant quantity, and that of

\footnotetext{
* Corresponding author. Tel.: +7 3422466 514; fax: +7 3422371636 .

E-mail address: nataliya.skryabina@grenoble.cnrs.fr (N.E. Skryabina).
}

the $\sigma$-phase is approximately 6.5-7 [3] (or 5.76 from structure bond considerations [4]). Also the valence electron concentration (VEC) has been considered as a pertinent factor for the existence of a low temperature superconducting state [5].

\section{Experimental}

Before examining the impact of hydrogenation on the stability and the properties of a $\sigma$-phase material, we report on the synthesis of the compound $\left(\mathrm{Fe}_{53.8} \mathrm{Cr}_{46.2}\right)$. Our investigated alloy was prepared by melting under vacuum constituent elements of $3 \mathrm{~N}$-purity by using an induction furnace. The ingots were subsequently annealed at $1273 \mathrm{~K}$ for 3 days. Afterwards the material was cold-rolled to a final $1 \mathrm{~mm}$ thickness. Then, the transformation to a $\sigma$-phase compound was carried out by isothermal annealing the resulting slabs at $973 \mathrm{~K}$ under secondary vacuum. Next, the control of the transformation degree was checked first by X-ray diffraction, and second the material was characterized by ${ }^{57} \mathrm{Fe}$ Mössbauer spectrometry.

Loading rolled samples with hydrogen was performed electrochemically for no more than $1 \mathrm{~h}$, as determined by optimization of the parameters leading to the best rate of hydrogenation. The cathodic current density was monitored with reference to a specific measurement of the polarization curves in $0.5 \mathrm{M}$ $\mathrm{H}_{2} \mathrm{SO}_{4}$ as shown in Fig. 1. Such data were used to determine the Tafel slopes. Accordingly, the cathodic current density was fixed to $250 \mathrm{~A} / \mathrm{m}^{2}$. Measurements of microhardness by a standard method, X-ray diffraction analyzes using a PW 1720 Philips set up (wavelength $\lambda=\mathrm{Cu} \mathrm{K} \alpha$, backscattering graphite monochro- 


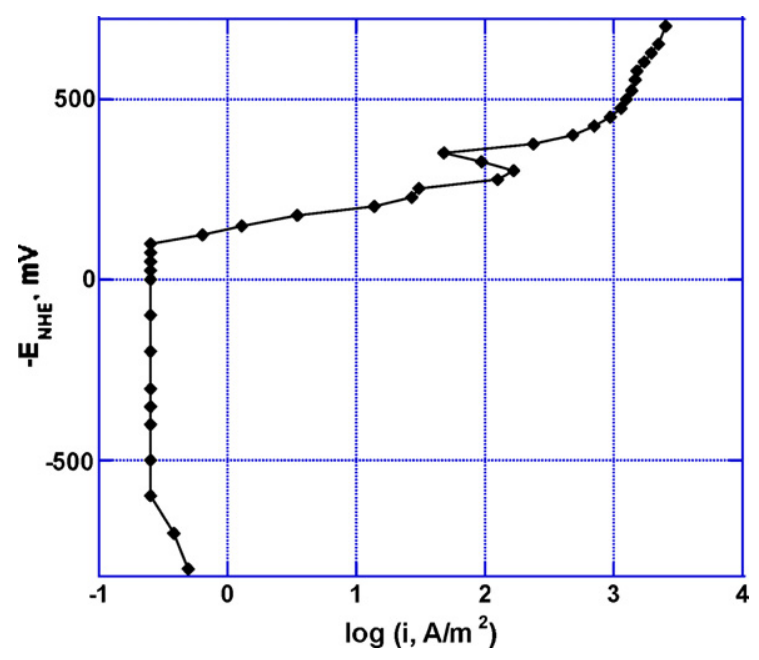

Fig. 1. Polarization curves of the $\mathrm{Fe}_{53.8} \mathrm{Cr}_{46.2}$ alloy in $0.5 \mathrm{M} \mathrm{H}_{2} \mathrm{SO}_{4}$ with $\mathrm{As}_{2} \mathrm{O}_{5}$ as a hydrogenation activator. From $\sim 750$ to $-370 \mathrm{mV}$ the trace corresponds to an anodic regime, from $\sim-370$ to $\sim-750 \mathrm{mV}$, the trace corresponds to a cathodic regime.

mator) and Barkhausen effect measurements according to a method described in [6], were undertaken immediately after achieving hydrogenation and 6 months later. The Mössbauer spectroscopy measurements were performed at room temperature 2 days after the hydrogenation, as well as at 10 and $4.2 \mathrm{~K}, 1$ month after loading with hydrogen. The ${ }^{57} \mathrm{Fe}$ Mössbauer spectra were recorded in transmission geometry using a standard spectrometer. $\gamma$-rays of $14.4 \mathrm{keV}$ were supplied by using a ${ }^{57} \mathrm{Co} / \mathrm{Rh}$ source. Moreover, fluorescence analysis with a scan step of $0.02 \AA$ was made to check a potential redistribution of the elements onto the surface before and after H-loading.

\section{Results and discussion}

\subsection{Structure and microstructure aspects}

It was shown earlier $[7,8]$ that $\mathrm{H}$-loading can induce in alloys a certain redistribution of metal elements. Such a phenomenon should be related to the increase of both internal stresses and of the effective diffusion coefficient of both metal and $\mathrm{H}$ atoms at hydrogenation. In the $\mathrm{Fe}-\mathrm{Cr}$ alloys, the $\sigma$-phase exists in a narrow range of composition. Following our previous studies loading with hydrogen was expected to lead to some potential disproportionation effects of the material, see for example, ref. [9], accounting here for to the restricted stability of the $\sigma$ phase. However, the $\sigma-\mathrm{Fe}_{53.8} \mathrm{Cr}_{46.2}$ alloy was demonstrated to be stable under hydrogenation since no new phase was evidenced as shown in Fig. 2. Hydrogenation of the alloy leads to (1) a shift of all diffraction lines towards smaller Bragg angles; consequently, cell parameters $a$ and $c$ of the tetragonal structure $\left(\mathrm{P}_{2} / \mathrm{mnm}\right)$ are significantly increased, (2) an significant increase of the width of selected series of the diffraction peaks. For comparison $a=8.742 \AA, c=4.567 \AA, V=349 \AA^{3}$ and $c / a=0.522$ for the starting alloy, and $a=8.829 \AA, c=5.290 \AA, V=403.6 \AA^{3}$ and $c / a=0.59$ for the H-loaded material. The increase of the volume due to the hydrogen insertion is $\Delta V \sim 54.5 \AA^{3}$ per unit cell, which is $\sim 15.6 \%$. The hydride composition can be approximated as $\mathrm{Fe}_{53.8} \mathrm{Cr}_{46.2} \mathrm{H}_{\sim 1.2}$ as normalized to the unit formula and taking for the "unit volume" of one hydrogen atom occu-
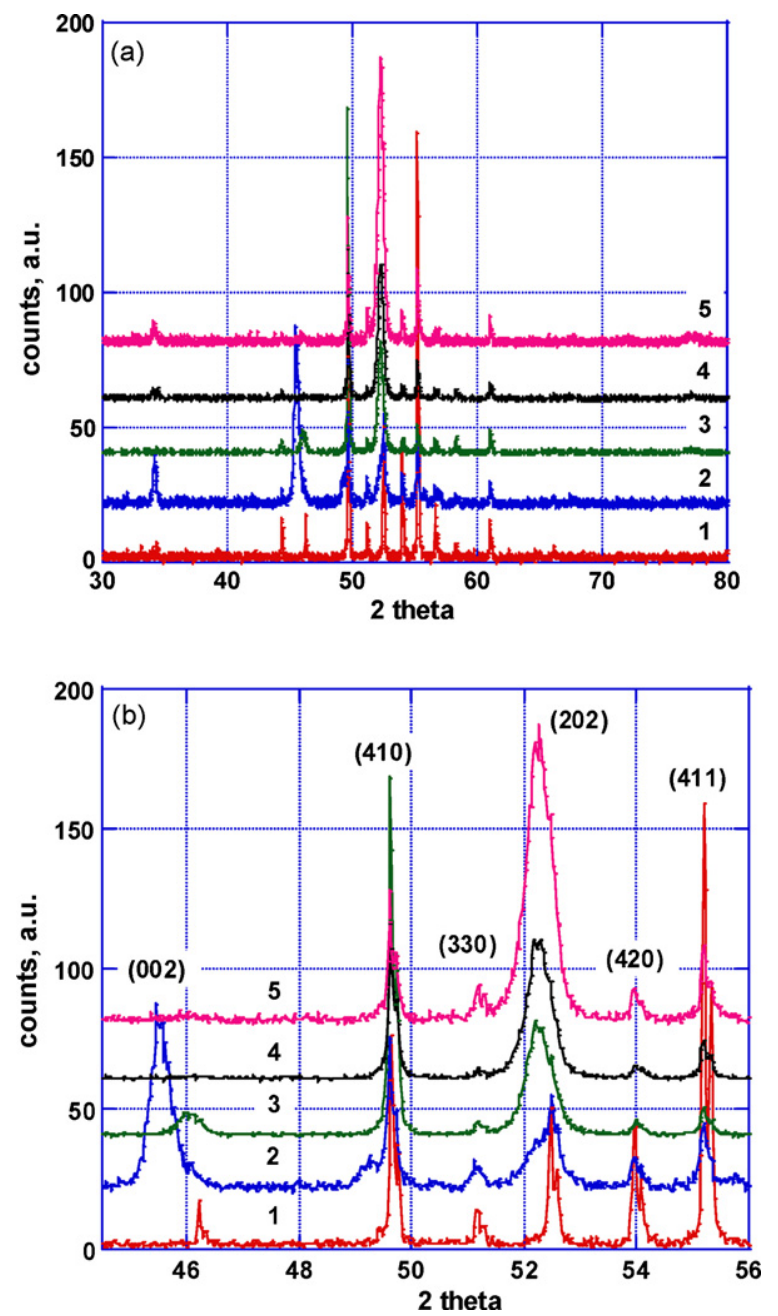

Fig. 2. (a) X-ray diffraction patterns of the $\mathrm{Fe}_{53.8} \mathrm{Cr}_{46.2}$ alloy and its hydride-1: initial state, 2-5 after H-loading: 2: immediately after, 3-4 days after, 4-11 days after, and 5-14 days after; (b) fragment of the diffraction patterns shown in (a) shown in a different scale to exhibit the broadening effect.

pying a tetrahedron in a metal system a value of $\Delta V_{\mathrm{H}} \sim 2.9 \AA^{3}$ [10]. This value appears quite reasonable in the light of what it was found for parent alloys and compounds such as $\mathrm{FeTi}, \mathrm{Cr}$, etc. $[11,12]$. However, after few days aging, the cell parameters were found to come back to their typical values characteristic of the sample in the initial state as shown in Fig. 3. Interestingly, the change of the structure parameters is not accompanied by a redistribution of metal elements of the alloy. The results of the Xray investigation and of fluorescence analysis confirm this fact in a good agreement with measurements of the Barkhausen effect. From this later technique which is very sensitive to, e.g. magnetic metal atom reordering, no evidence of typical "Barkhausen noise" was found before and after hydrogenation as well after dehydrogenation.

We would like to underline that from the X-ray diffraction patterns, no valuable crystal structure refinement can be performed for several reasons, unfortunately. Firstly, because the structure is not stoichiometric and quite different proportions of $\mathrm{Cr}$ and $\mathrm{Fe}$ atoms are related to the five different crystallographic sites, whose position parameters have also to be accounted 


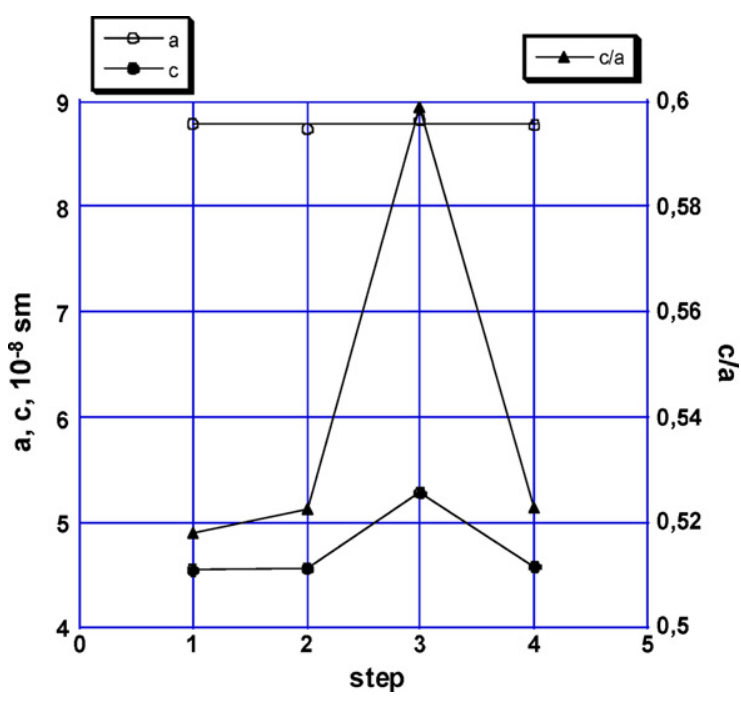

Fig. 3. Unit cell parameters of the $\mathrm{Fe}_{53.8} \mathrm{Cr}_{46.2}$ alloy. Steps-1: data from [14], 2: initial state, 3: just after hydrogenation, 4-6 months aging after hydrogenation.

for. Furthermore, in the parent hydrogenated samples and with reference to Fig. 2a-2 to Fig. 2a-5, one has to consider the corresponding $\mathrm{H}$-site occupation factors as well as the positional parameters. Secondly, the initial $\sigma$-phase sample in for of a slab was delivered highly textured since it had been cold-rolled prior to the isothermal annealing in order to get the appropriate form for performing the electrochemical charging and following diffraction experiments. Consequently, the diffraction pattern analysis must also account for the marked texture effect. Thirdly, the very anisotropic unit cell expansion leads to distinguished broadening effects of different diffraction lines, probably due to an anisotropic distribution of the interstitial sites occupied by hydrogen atoms. It appears that the $(h k 0)$ types of lines are little or not at all affected by the development of structural stacking defects, contrary to the [0 01$]$ or other close directions. It is worth concluding that the hydrogen insertion scheme has a net 2D character, but it results in that various profile coefficients $(U$, $V, W)$ should be considered for structural refinement, as well.

At present, neutron diffraction experiments are planned to overcome well the question of crystal structure refinements, making profit of the large differences in scattering lengths of chromium, iron and deuterium, the later used instead of hydrogen.

Fig. 4 reveals that the microhardness (HV, Vickers) decreases from $\sim 1100$ to $1200 \mathrm{~kg} / \mathrm{mm}^{2}$ before, to approximately $750-780 \mathrm{~kg} / \mathrm{mm}^{2}$, after hydrogenation. In both cases these values depend weakly on the applied stress, thus after hydrogenation, the microhardness $\mathrm{HV}$ does not result from the hydrogen embrittlement. The regime of electrochemical Hloading was performed in such a way that initially the hydrogen permeation resulted in the formation of a solid solution as experimented before [13]. In earlier experiments realized on iron and iron-based alloys, it was found that at room temperature (RT) hydrogen evacuates from the solid solution phase of the loaded samples within 3 days after the loading has been completed, even if no cracks were created during hydrogenation.

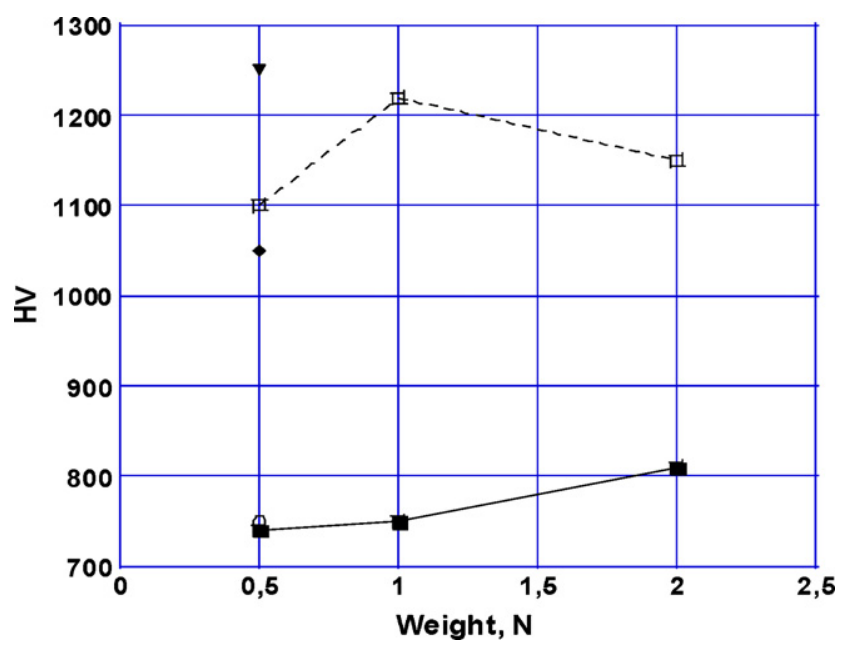

Fig. 4. Vickers microhardness HV measurements vs. the stress applied. Symbols: $(\boldsymbol{\nabla})$ data from [15], $(\bigcirc)$ data from [16], ( $)$ data from [17] for $\mathrm{Fe}-\mathrm{Cr}$ alloys. For the present alloy $\mathrm{Fe}_{53.8} \mathrm{Cr}_{46.2}:(\square)$ initial state, (ם) immediately after hydrogenation. The lines are guides to the eyes.

\subsection{Magnetic characteristics}

Mössbauer spectra were recorded first at RT 2 days after Hloading, then at $10 \mathrm{~K} 1$ month later. In both cases, no significant difference could be evidenced between the $\mathrm{H}$-loaded and $\mathrm{H}$-free samples. However, in the spectrum recorded at $4.2 \mathrm{~K}$, a weak but visible change of the hyperfine magnetic field were found which can be related to slight modifications of a Fe-site spin-density. Such spin-density changes usually reflect chemical, topological or structural modifications in the Fe-site neighborhood. The spectra were fitted in terms of a distribution of the hyperfine field method, and the resulting from this procedure histograms are shown in Fig. 5. It is to be pointed out that a main difference, even if weak, occurs in the range of small fields $(2-3 \mathrm{~T})$, i.e. where is situated the main peak of the hyperfine field distribution. In the $\sigma$-phase, the five different site positions which are occupied both by $\mathrm{Fe}$ and $\mathrm{Cr}$ atoms in different proportions, are denoted 2a, 4f, $8 \mathrm{i}, 8 \mathrm{i}$ and $8 \mathrm{j}$. However, due to a lack of stoichiometry, instead of five specific hyperfine fields (or less) characteristic of the five crystallographic site, one obtains a broad distribution of fields. As follows from Fig. 5, the main effect of hydrogen loading is restricted to the fields close to $2-3 \mathrm{~T}$, which probably means that no more than one $\mathrm{Fe}$-site with a $\mathrm{Cr}$-rich coordination remains affected by the hydrogen insertion. The maximum distribution peak is shifted towards higher hyperfine fields, meaning that the magnetic moment of the involved iron site is increased. Rescaling the hyperfine field (in T) to magnetic moment (in $\mu_{\mathrm{B}}$ ) based on ref. [18], an estimate of the magnetic moment can be made as $0.15 \mu_{\mathrm{B}}$ in the alloy and as $0.2 \mu_{\mathrm{B}}$ in the hydride. Furthermore, there is also a change in the maximum field (top the distribution), which increases from 16 to $16.5 \mathrm{~T}$. This can be related to the mean volume expansion on hydrogenation leading to a reinforcement of the overall magnetization. On the other hand, there is no measurable effect of the hydrogenation on the isomer shift, which is a measure of the charge density at Fe-site. However, for magnetic metals and alloys, a change in the charge 

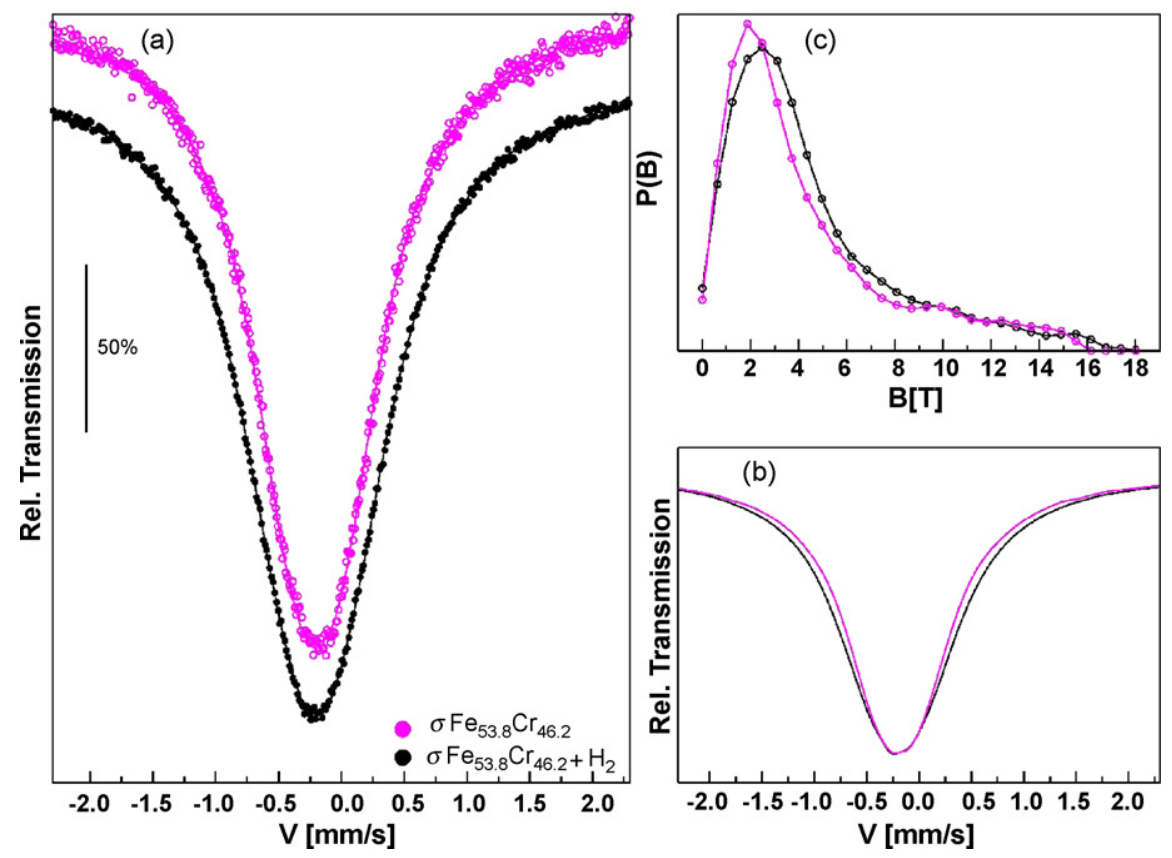

Fig. 5. (a) ${ }^{57} \mathrm{Fe}$ Mössbauer spectra recorded at $4.2 \mathrm{~K}$ on the hydrogen-free and hydrogen initially charged samples, (b) the best-fit spectra of (a), and (c) the magnetic hyperfine field distributions curves obtained from the spectra. Gray colour stays for the hydrogen-free and black colour for the hydrogen-charged samples.

density is usually one order magnitude smaller than that in the spin-density.

There is an indirect correlation between the results of X-ray and Mössbauer spectroscopy analysis. Hydrogen atoms should occupy preferentially some of the interstitial sites in the sigmaphase unit cell. Typically, all the interstitial sites have a fourfold metal coordination since the sigma-phase belongs to the class of tetrahedrally close-packed structures [19]. In principle, for the $\mathrm{Fe}-\mathrm{Cr}$ alloy, the sites exhibiting the most electro-attractive character should be the tetrahedra dominantly formed with $\mathrm{Cr}$ atoms $2 \mathrm{Cr}(4 \mathrm{f})-2 \mathrm{Cr}(8 \mathrm{j})$. However, the number of such sites is not large enough to explain the maximum amount of hydrogen atoms $H_{\max }$ per unit cell as deduced from the X-ray analysis $\left(\sim 0.27\right.$ instead of $\left.H_{\max } \sim 1.2\right)$. Consequently, 3Cr-Fe sites must be also taken into account, e.g. $2 \mathrm{Cr}(4 \mathrm{f})-\mathrm{Cr}(8 \mathrm{j})-\mathrm{Fe}(2 \mathrm{a})$ or $2 \mathrm{Cr}(8 \mathrm{j})-\mathrm{Cr}(4 \mathrm{f})-(\mathrm{Fe}, \mathrm{Cr})(8 \mathrm{i})$, etc. At the same time one must account for both the scheme of $\mathrm{Fe}$ and $\mathrm{Cr}$ atom distribution on specific metal sites and the exclusion rule for $\mathrm{H}$ atoms simultaneously occupying those tetrahedra sharing a common triangular face [20]. However, since the above considered sites as possible ones for the hydrogen insertion are directly stacked along the $z$-axis, it is anticipated that the $c$ cell parameter must increase predominantly as found experimentally. Furthermore, the most sensitive site to hydrogenation in terms of the hyperfine field should be the $\mathrm{Fe}(8 \mathrm{i})$ sites. But a more specific technique such as neutron diffraction must be used, firstly to determine the hydrogen site occupation scheme and secondly to evaluate its impact on the $\mathrm{Fe}$ magnetic moments. In situ H-loading neutron diffraction experiments are planned, also we expect to record concomitant Mössbauer spectroscopy data on freshly loaded samples, before the rapid desorption occurs, as reported here.

\section{Conclusions}

Based on the results obtained in this study we can conclude that hydrogen could be successfully introduced into the $\sigma$-phase of a $\mathrm{Fe}-\mathrm{Cr}$ compound. Its concentration estimated from the increase of the unit cell volume just after loading was equal to $\sim 1.2 \mathrm{H}$ per formula unit. The observed cell expansion was found to be very anisotropic and accompanied by creation of numerous defects stacked mostly along the [ $\left[\begin{array}{ll}0 & 0\end{array}\right]$ direction. The hydride formed turned out to be very unstable which had prevented a more detailed characterization in terms of other physical properties.

\section{Acknowledgements}

B.F.O. Costa wishes to acknowledge financial support from FCT/POCTI, co-financed by the FEDER European Community fund (project POCTI/CTM/40759/2001). One of us (NS) was partly supported by the ECO-NET scheme of exchange funded by the French Ministry of Foreign Office (grant no. 08133RA).

\section{References}

[1] D. Fruchart, J.L. Soubeyroux, S. Miraglia, S. Obbade, G. Lorthioir, F. Basile, C. Colin, F. Faudot, P. Ochin, A. Dezellus, Zeit. fur Phys. Chem. 237 (1993) 225.

[2] L.V. Spivak, N.E. Skryabina, D. Fruchart, L. Cagnon, J. Alloys Compd. 404-406 (2005) 550.

[3] P. Greenfield, P.A. Beck, Trans. AIME 200 (1954) 253; P. Greenfield, P.A. Beck, Trans. AIME 205 (1954) 265.

[4] B.G. Bergman, D.P. Shoemaker, Acta Crystallogr. 7 (1954) 857.

[5] B.W. Roberts, in: J.H. Westbrook (Ed.), Intermetallic Compounds, Krieger Publishing Company, N.Y., 1977, p. 581. 
[6] D. Fruchart, N. Skryabina, L. Spivak, J. Alloys Compd. 383 (2004) 180.

[7] N. Skryabina, D. Fruchart, L. Spivak, J. Metastable Nanocryst. Mater. 20-21 (2004) 517.

[8] A.M. Avdukhina, A.A. Katsnelson, G.P. Revkevich, Acta Crystallogr. 44 (1) (1999) 1.

[9] V.A. Goltsov, D. Fruchart, S.B. Rybalka, V.A. Didus, in: V.A. Goltso (Ed.), Progress of Hydrogen Treatment of Materials, Kassiopaya Dontesk-Coral Gables IHEA, 2001, ISBN 966-7418-71-5.

[10] H. Peisl, in: G. Alefeld, J. Völk (Eds.), Hydrogen in Metals I, Springer, Berlin, Heidelberg, Top. Appl. Phys. 28 (1978) 53.

[11] K. Yvon, P. Fischer, in: L. Schlapbach (Ed.), Hydrogen in Intermetallic Compounds I, Springer, Berlin, Heidelberg, 1988, p. 87.
[12] Y. Fukai, M. Mizutani, Mater. Trans. 43 (2002) 1079.

[13] N. Skryabina, D. Fruchart, L. Cagnon, A.V. Shelyakov, J. Metastable Nanocryst. Mater. 570 (2008) 65.

[14] E.O. Hall, S.H. Algie, Metall. Rev. 11 (1966) 61.

[15] B.F. Costa, S.M. Dubiel, Phys. Status Solidi A 139 (1993) 83.

[16] G.V. Smith, Iron Age 30 (1950) 63.

[17] G. Pomey, P. Bastien, Revue de Métallurgie L111 (2) (1956) 147.

[18] J. Cieślak, B.F.O. Costa, S.M. Dubiel, M. Reissner, W. Steiner, J. Phys. Condens. Matter 17 (2005) 2985.

[19] F.C. Frank, J.S. Kasper, Acta Crystallogr. 11 (1958) 184; F.C. Frank, J.S. Kasper, Acta Crystallogr. 12 (1959) 483.

[20] D.P. Shoemaker, C.B. Shoemaker, J. Less-Common Met. 68 (1979) 43. 